\title{
A Road Condition Classification Algorithm for a Tire Acceleration Sensor using an Artificial Neural Network
}

\author{
Hyeong-Jun Kim ${ }^{1}$, Jun-Young Han ${ }^{1}$, Suk Lee ${ }^{1}$, Jae-Ryon Kwag ${ }^{2}$, Min-Gu Kuk ${ }^{2}$, In-Hyuk Han ${ }^{2}$ \\ and Man-Ho Kim ${ }^{3, *}$ \\ 1 School of Mechanical Engineering, Pusan National University, Pusan 46241, Korea; \\ jun0423@pusan.ac.kr (H.-J.K.); jyhan@pusan.ac.kr (J.-Y.H.); slee@pusan.ac.kr (S.L.) \\ 2 Performance Research Team, NEXEN Tire, Yangsan-si 50592, Korea; jaery@nexentire.com (J.-R.K.); \\ mg.guk@nexentire.com (M.-G.K.); InhyukHan@nexentire.com (I.-H.H.) \\ 3 Division of Automotive Engineering, Dong-eui Institute of Technology, Pusan 47230, Korea \\ * Correspondence: mhkim@dit.ac.kr; Tel.: +82-51-860-3261
}

Received: 29 January 2020; Accepted: 26 February 2020; Published: 28 February 2020

check for updates

\begin{abstract}
The automotive industry is experiencing a period of innovation, represented by the term CASE (connected, autonomous, shared, and electric). Among the innovative new technologies for automobiles, intelligent tire (iTire) collects road surface information through sensors installed inside a tire and informs the driver of the road conditions. iTire can promote safe driving. Various kinds of research on iTire is ongoing, and this paper proposes an algorithm to determine the road surface conditions while driving. Specifically, we have proposed a method for extracting the feature points of a frequency band, by converting acceleration data collected by sensors through fast Fourier transform (FFT) and determining road surface conditions via an artificial neural network. Lastly, the applicability of the algorithm was verified.
\end{abstract}

Keywords: Intelligent tire (iTire); tire acceleration sensor; road condition classification; tire pressure monitoring system (TPMS); artificial neural network

\section{Introduction}

The automotive industry is experiencing a period of innovation, represented by the term CASE (connected, autonomous, shared, and electric). This term symbolizes the application of technologies from various fields to automobiles [1-3]. Considerable effort has also been directed toward improving driving stability and convenience by utilizing various vehicle technologies, such as sensors and wired/wireless communications [4-6]. Tires, which are in direct contact with the road surface, greatly affect the stability of the vehicle. As a technology that improves vehicle stability and driver convenience, tire-pressure monitoring systems (TPMSs) measure tire pressure and relay that information to the driver [7]. TPMS has been mandatory for automobiles sold in the United States since 2007, and in Korea since 2015 [8,9].

TPMS can prevent tire-related accidents $[10,11]$. However, it is also important that the driver is aware of road conditions, such as whether the surface is normal, unpaved, wet, snow-covered, or icy $[12,13]$. Given that tires are in direct contact with the road surface, information regarding not only tire-related, such as pressure, temperature, wear condition, and tread depth, but also information on the degree of road surface friction and pavement condition, can be obtained [14]. Tires that collect such information through an internal sensor and relay data on the road surface conditions to the driver are called intelligent tires (iTires). Cars equipped with iTire technology can determine the road 
condition on behalf of the driver, which may not be known by the driver, and ensure adequate steering and braking, thereby promoting safer driving $[15,16]$.

Tire-based methods for sensing and classifying road conditions include utilizing the slip ratio (given by the speed ratio between the driving and driven wheels of a vehicle), applying information acquired visually, and using an ultrasonic sensor. This study used data on vibrations sensed by the tires. Kanwar et al. determined road surface conditions through a fuzzy logic-based method, in which the maximum frictional force was calculated using tire load and slip data [17]. However, that study used simulation data rather than data obtained in a real vehicle environment, so further studies are required for validation. Niskanen et al. conducted a study to distinguish between two road surfaces with different friction conditions by attaching an acceleration sensor to the inside of a tire [18]. Their road condition classification method can be used to measure acceleration within the leading edge section of the tire, i.e., the section before the sensor contacts the ground, in the frequency domain. However, the road surface has to be classified as either concrete or ice, where these surface types show large differences. Hanatsuka et al. classified road surface conditions using a support vector machine (SVM) learning technique [19]. Their method provided high road condition classification accuracy when used with tires of various sizes, but it is difficult to apply this method in real time due to the large computational load. In a subsequent study, a road condition classification method that can be applied in real time was introduced based on high-speed kernel computation [20]. However, a high-frequency acceleration sensor is required (5 kHz or higher), which leads to high energy consumption and costs.

In this paper, we describe the iTire system and propose an algorithm for determining road conditions using data from an acceleration sensor installed inside a tire. Specifically, our algorithm involves extracting the feature points of the frequency band, by converting the collected acceleration data through fast Fourier transform (FFT) and then applying an artificial neural network (ANN) analysis. Road conditions were classified as dry, wet, or rough. Finally, we verified the applicability of the algorithm using acceleration data accrued by the sensor for an actual vehicle.

This paper consists of five sections. Section 2 describes the iTire system. Section 3 provides the method for extracting feature points and an algorithm for determining road surface conditions using ANN analysis. Section 4 describes the experimental environment and the verification results. The final section reports the conclusions and future research plans.

\section{2. iTire System}

In general, iTires utilize the output of an acceleration sensor attached inside the tire to determine road conditions, which are relayed to the driver. The communication modality for iTires is usually wireless, because the sensor attached inside the rotating tire cannot be wired to the processing unit installed in the main body of the vehicle. Among the various wireless communication methods, Bluetooth is the most widely used because of its reliability and convenience. Figure 1 shows a schematic of the iTire system, including the accelerometer, the pathway for transmission of the acceleration data, and the road condition classification module that receives the data.

The acceleration sensor can measure up to $200 \mathrm{~g}$ on three axes and outputs data at a $1 \mathrm{kHz}$-sampling rate; it is equipped with a Bluetooth transmitter. The sensor can convert measured analog values into digital values and then into Bluetooth (wireless) format. Bluetooth 4.2 can transmit up to a distance of $300 \mathrm{ft}(91 \mathrm{~m})$, at a transmission rate of up to $1 \mathrm{Mbps}$. Bluetooth 5.0, which was recently developed, can achieve a transmission range and rate of $1200 \mathrm{ft}(366 \mathrm{~m})$ and $2 \mathrm{Mbps}$, respectively.

The road condition classification system consists of a Bluetooth receiver (which receives the sensor information), a function that processes the received acceleration sensor values through FFT, and a function to determine the road conditions using ANN analysis. The data periodically transmitted from the acceleration sensor via Bluetooth are received by the Bluetooth receiver and transferred to a signal pre-processing unit that performs feature extraction (by converting the measured tire acceleration sensor values via FFT). Finally, ANN analysis determines the road surface conditions based on the features generated by the signal pre-processing unit. 


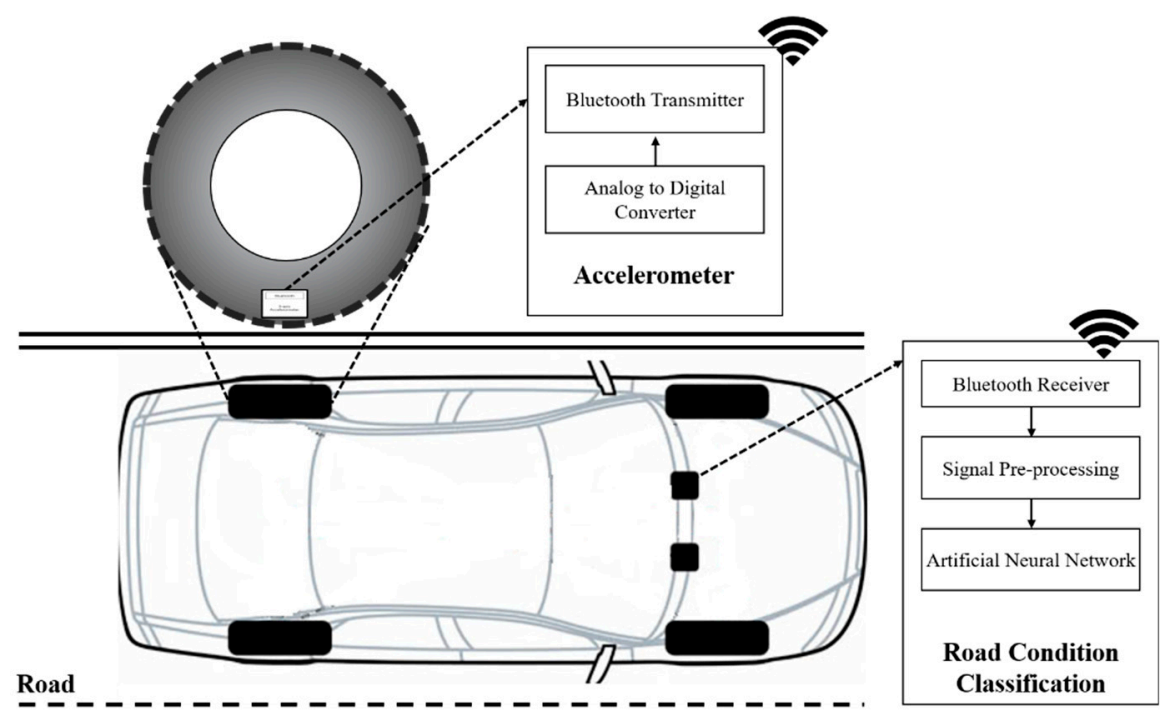

Figure 1. Schematic of the iTire and road condition classification algorithm.

\section{Road Condition Classification Algorithm}

Figure 2 illustrates the algorithm used by the iTire system, which involves data acquisition via the acceleration sensor, processing of the signal using FFT, and determining the road conditions using ANN analysis. After initialization, the road condition classification algorithm acquires $\mathrm{K}$ signal values from the acceleration sensor. Determining the road condition is made more difficult by vibrations that increase with the shock applied to the tire, and by the noise generated in a real road environment. $\mathrm{K}$ was determined through trial and error and was acquired 2000 times over $2 \mathrm{~s}$ at $1 \mathrm{kHz}$.

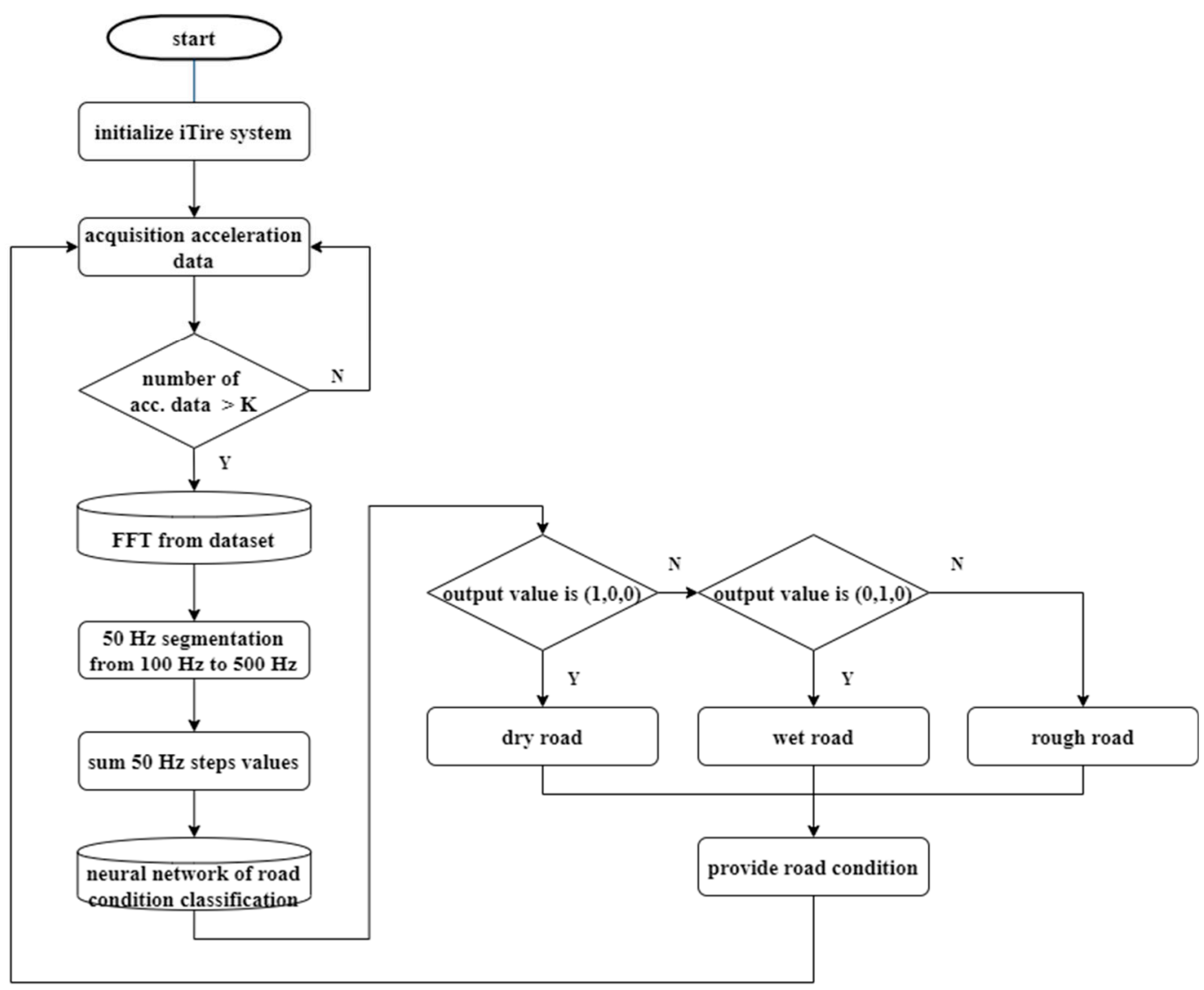

Figure 2. Road Condition Classification Algorithm of the iTire System. 
Once $\mathrm{K}$ acceleration sensor values are acquired, they can be processed via FFT. The values are divided into frequency bands, with segmentation at $50 \mathrm{~Hz}$ intervals from 100-500 Hz. The $0 \mathrm{~Hz}$ band is a direct current component providing velocity information about the vehicle. Values at $1-100 \mathrm{~Hz}$ are considered to be unsuitable for determining the road condition and are thus excluded from the analysis. The power spectrum values of each 100-500 Hz segment are summed. Figure 3 shows the results of summing each band, in terms of the road conditions (dry, wet, or rough) and velocity (40,60 or $80 \mathrm{kph}$ ). In the figure, the value for the $0 \mathrm{~Hz}$ band indicates the velocity of the vehicle sufficiently well regardless of the road conditions, although there is a difference in that value according to the road conditions.

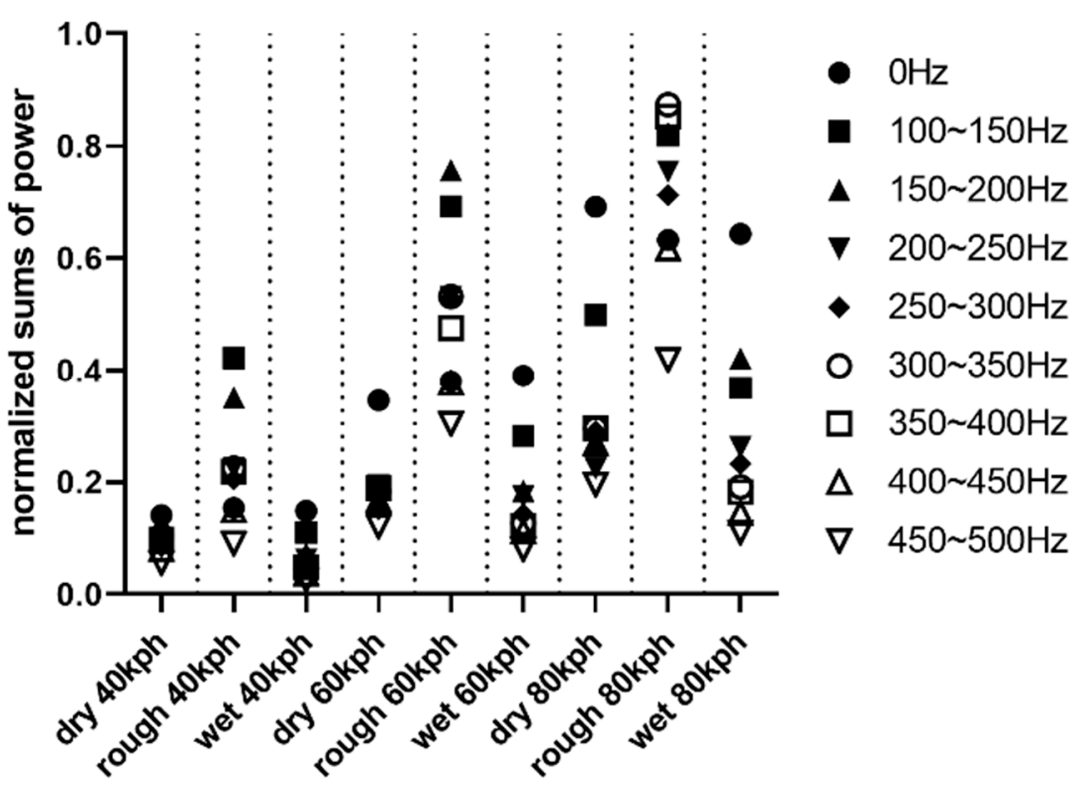

Figure 3. Distribution of normalized sums of power.

The nine values obtained at $0 \mathrm{~Hz}$, and at each interval in the range $100-500 \mathrm{~Hz}$, were normalized so that they had a value between 0 and 1 . These data were then used as input for the ANN, which determined the road condition (dry, wet, or rough). Back-propagation algorithm [21], an optimization technique, was used as the ANN learning algorithm. The hidden layer of the ANN consisted of six hidden nodes. The final output value of the ANN corresponded to the road conditions (dry, wet, or rough); the ANN was trained to converge to 1 for the likely road condition and 0 for the other road conditions. In total, $40 \%$ of the driving data were used for learning, and the remaining $60 \%$ for testing the results of the learning.

\section{Discussion Performance Evaluation of the Road Condition Classification Algorithm}

Acceleration sensor values were collected from a dedicated test site (proving ground, PG) that simulated various road conditions. These values were used to evaluate the learning performance and road condition classification algorithm. Figure 4 shows the experimental setup of the iTire system for collecting acceleration data. Figure 4 a shows the acceleration sensor board, consisting of a sensor module, an analog-digital converter, and a Bluetooth transmitter. Figure $4 \mathrm{~b}$ shows the road condition classification module, consisting of a Bluetooth receiver, a signal pre-processing unit, and an ANN. Figure $4 \mathrm{c}$ shows the acceleration sensor board attached to the inside of a tire, and Figure $4 \mathrm{~d}$ shows the power supply module (center of the wheel). The power supply provides the power needed to operate the acceleration sensor, for which a 7.4 V, $2000 \mathrm{~mA} \mathrm{Li}$-ion battery was used.

To acquire sensor data, three conditions (dry, wet, and rough road surfaces) were set up in the dedicated PG. Here, dry refers to a typical asphalt road surface, wet to a road surface on which a water film about $8 \mathrm{~mm}$ deep was maintained, and rough to a section paved with rough asphalt. In total 426,000 data points were collected through repeated driving on straight road surfaces under various 
surface (dry, wet, rough) and velocity $(40,60,80 \mathrm{kph})$ conditions. Table 1 shows the amount of data collected for evaluating the performance of the road condition classification algorithm. Because the dedicated PG had short wet and rough sections, fewer data were collected per pass under those conditions compared to the dry section. Also, as speed increased, the passing time was shorter, so fewer data were collected per pass.

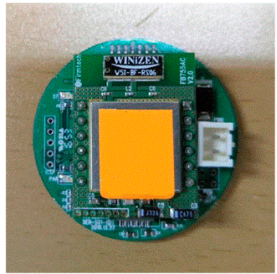

(a)

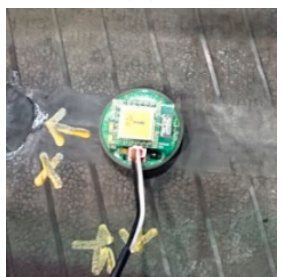

(c)

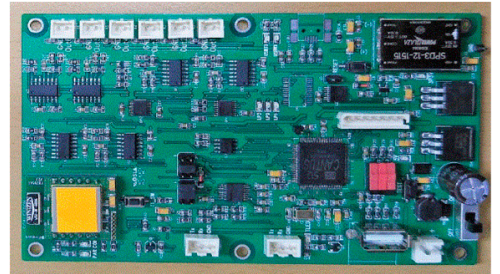

(b)

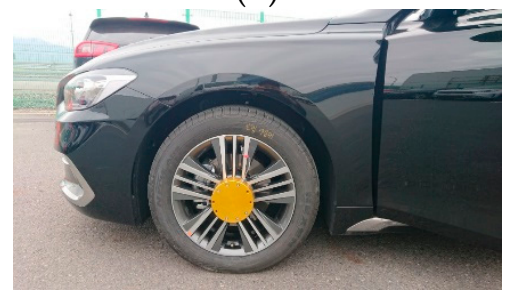

(d)

Figure 4. iTire system experimental setup: (a) sensor board, (b) classification module, (c) sensor board attached to the inside of a tire, and (d) battery module (at wheel center).

Figure 5 shows example acceleration sensor data for the dry road, before they were subjected to FFT processing. In the figure "Leading edge" indicates the point where the acceleration sensor comes into contact with the road surface, "Contact" denotes the section maintaining road contact, and "Trailing edge" indicates the point where road contact ends. Here, the $x$-axis indicates the front and rear parts of the vehicle, and the $y$-axis the left and right parts. The z-axis is the vertical dimension. In Figure 5a, the acceleration on the $x$-axis shows that the output decreased at the leading edge and returned to the original output waveform after passing the trailing edge. Acceleration on the y-axis in Figure $5 b$, and on the z-axis in Figure 5c, show that the output was lowest at the leading edge and highest at the trailing edge. Acceleration on the $\mathrm{x}$ - and y-axes showed similar characteristics, while acceleration on the $\mathrm{z}$-axis showed different values. These acceleration sensor values were used as input for determining the road surface condition via ANN analysis after FFT processing.

Table 1. Number of acceleration data points measured while driving in the proving ground (PG).

\begin{tabular}{ccc}
\hline Road condition & Velocity (kph) & Number of data points \\
\hline \multirow{2}{*}{ Dry } & 40 & 144,000 \\
& 60 & 72,000 \\
\multirow{2}{*}{ Wet } & 80 & 72,000 \\
& 40 & 20,800 \\
& 60 & 15,600 \\
Rough & 80 & 15,600 \\
& 40 & 43,000 \\
& 60 & 26,875 \\
& 80 & 16,125 \\
\hline
\end{tabular}

In this paper, the relationship between the acceleration data measured through iTire and the road surface condition was modeled using MLP. Nine values obtained through pre-processing (at $0 \mathrm{~Hz}$, and then at each $50 \mathrm{~Hz}$ interval from 100-500 Hz, where these data were summed) and a bias value were selected as input variables for the input layer. The hidden layer had six nodes, and the final layer 
output one of three road conditions (dry, wet, or rough). To enable the edge connecting the nodes to learn the optimal weight, the learning rate was set to 0.05 and the target error to 0.01 . Sigmoid was chosen as the activation function. About $40 \%$ of the collected data were used for learning, and the remaining $60 \%$ were used as input to verify performance.

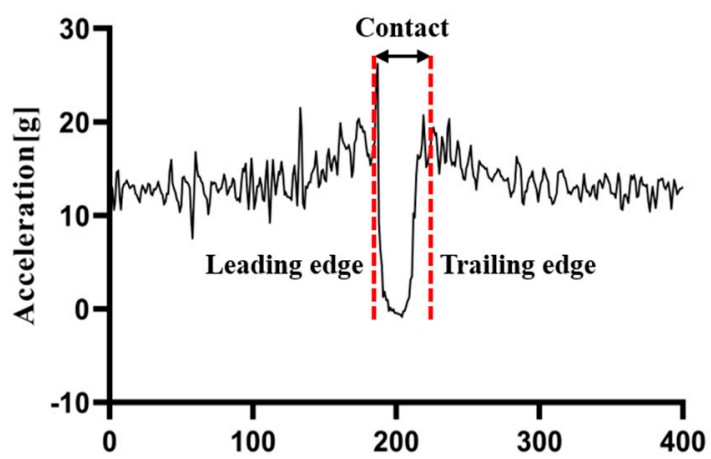

(a)

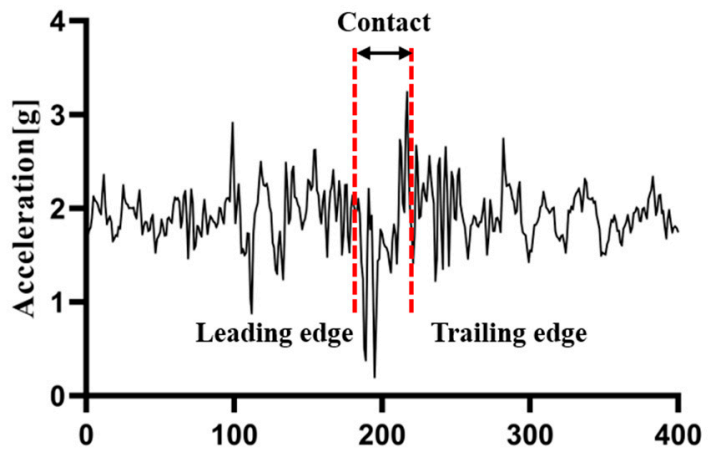

(b)

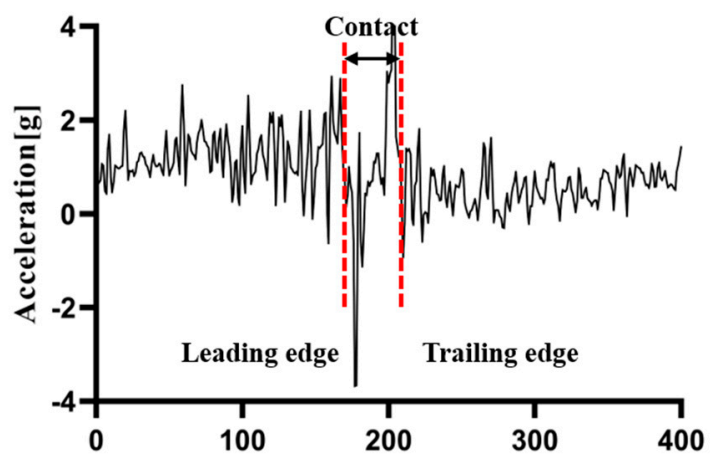

(c)

Figure 5. Example acceleration signal under the dry road condition: (a) x-axis acceleration signal, (b) y-axis acceleration signal, and (c) z-axis acceleration signal.

Table 2 shows the performance evaluation results of the road condition classification algorithm. As the table shows, performance was evaluated based on at least 32,000, and up to 160,000, acceleration sensor values, depending on the road conditions. The target value representing the road surface condition was set to 1 . The target error, which was the basis of the road classification, was defined as the value obtained after subtracting the output value of the ANN from the target value. In one dataset for the dry road condition (2000 data points), the target error was calculated as $0.3062(=1-0.6938)$, which we determined to be a recognition error (where, in this paper, the acceptance criterion for classifying a road surface condition was an output value of more than 0.9 ). The reason for this large error value is not clear, but it is presumed that some of the driving occurred on another road condition during the course of the experiment, or that a mistake was made during data processing. 
Since an error occurred in 1 of 80 data sets (160,000 data points) under the dry road condition, the road condition classification accuracy was $98.75 \%$. No error occurred under wet or rough road conditions, therefore their accuracies were $100 \%$.

Overall, the road condition classification algorithm of the iTire system proposed herein showed excellent performance. Therefore, it is expected that the proposed road condition classification algorithm could be applied to a real vehicle, thereby improving driving safety.

Table 2. Number of acceleration data points measured while driving in the PG.

\begin{tabular}{cccc}
\hline & Dry & Wet & Rough \\
\hline Number of data points & 160,000 & 32,000 & 54,000 \\
Average target error & 0.008162 & 0.009454 & 0.004381 \\
Maximum target error & 0.3062 & 0.04632 & 0.006871 \\
Classification accuracy & $98.75 \%$ & $100 \%$ & $100 \%$ \\
\hline
\end{tabular}

\section{Conclusions}

This paper proposed an algorithm that can determine the road surface condition of a driving vehicle using an acceleration sensor attached to the inside of a tire. Specifically, a method was proposed for generating an input to an ANN after applying FFT. The algorithm determines the road surface condition using the acceleration sensor values extracted from the vehicle environment. Its applicability was verified, based on which the following conclusions can be drawn.

First, it was confirmed that the iTire system can be functionally implemented using a sensor board, a classification module, and a power supply module. Bluetooth wireless communication was confirmed as being suitable for collecting sufficient acceleration sensor data to determine the road conditions.

Second, it was confirmed that the acceleration sensor data could be used to determine the road surface conditions via signal processing. Specifically, the acceleration sensor output was divided into signals representing velocity and road surface condition.

Third, the proposed method for determining road surface conditions based on ANN analysis was highly accurate, suggesting its practical applicability to a real vehicle.

However, in this paper, only three kinds of road conditions (dry, wet, rough) were considered, therefore further studies are needed to classify more diverse environments, such as icy or snow-covered roads. Thus, more data are required, as well as research on more advanced learning processes, such as deep learning. Finally, further research on the proposed iTire system is necessary to achieve sustainability, via power supply methods such as energy harvesting.

Author Contributions: Conceptualization and writing-original draft, H.-J.K.; experiments and formal analysis, H.-J.K.; J.-Y.H.; J.-R.K.; M.-G.K.; I.-H.H.; supervision and writing-review and editing, S.L.; M.-H.K. All authors have read and agreed to the published version of the manuscript.

Acknowledgments: This research was supported by collaboration industry-academic research project with Nexen Tire Corporation and Academic Research Grant of 2019 Dong-eui Institute of Technology.

Conflicts of Interest: The authors declare no conflict of interest.

\section{References}

1. Moon, J.Y.; Bae, I.; Kim, S. An Inverse Vehicle Model for a Neural-Network-based Integrated Lateral and Longitudinal Automatic Parking Controller. Electronics 2019, 8, 1452-1467. [CrossRef]

2. Pevec, D.; Babic, J.; Podobnik, V. Electric Vehicles: A Data Science Perspective Review. Electronics 2019, 8, 1190-1220. [CrossRef]

3. Uhlemann, E. Introducing Connected. Veh. IEEE Veh. Technol. Mag. 2015, 10, $23-31$.

4. Guan, K.; Ai, B.; Peng, B.; He, D.P.; Li, G.K.; Yang, J.Y.; Zhong, Z.D.; Kürner, T. Towards Realistic High-Speed Train Channels at 5G Millimeter-Wave Band Part I: Paradigm, Significance Analysis, and Scenario Reconstruction. IEEE Trans. Veh. Technol. 2018, 67, 9112-9128. [CrossRef] 
5. Hu, Q.; Luo, F. Review of Secure Communication Approaches For In-Vehicle Network. Int. J. Automot. Technol. 2018, 19, 879-894. [CrossRef]

6. Doecke, S.; Grant, A.; Robert, W.G. The Real-World Safety Potential of Connected Vehicle Technology. Traffic Inj. Prev. 2015, 16, 531-535. [CrossRef] [PubMed]

7. Wang, J.; Song, F. On-Chip Integration of Pressure Plus 2-Axis (X/Z) Acceleration Composite TPMS Sensors with a Single-Sided Bulk-Micromachining Technique. Micromachines 2019, 10, 473. [CrossRef] [PubMed]

8. Velupillai, S.; Guvenc, L. Tire Pressure Monitoring [Applications of Control]. IEEE Control. Syst. Mag. 2007, 27, 22-25. [CrossRef]

9. Alexander, O.; Salem, E.; Chakhar, B.; Brown, D. An innovative decision rule approach to tire pressure monitoring. Expert Syst. Appl. 2019, 124, 252-270.

10. Robbems, W.B. Understanding Automotive Electronics: An Engineering Perspective, 8th ed.; Butterworth-Heinemann: Oxford, UK, 2017.

11. Lee, S.B.; Kim, D.H. Durable and Sustainable Strap Type Electromagnetic Harvester for Tire Pressure Monitoring System. J. Magn. 2013, 18, 473-480. [CrossRef]

12. Matsuzaki, R.; Keating, T.; Todoroki, A.; Hiraoka, N. Rubber-based strain sensor fabricated using photolithography for intelligent tires. Sens. Actuators Phys. 2008, 148, 1-9. [CrossRef]

13. Lee, H.J.; Taheri, S. Intelligent Tires? A Review of Tire Characterization Literature. IEEE Intell. Transp. Syst. Mag. 2017, 9, 10. [CrossRef]

14. Hariri, H.; Kim, J.; Kim, W.S.; Frechette, L.G.; Masson, P. Performance validation of printed strain sensors for active control of intelligent tires. Appl. Acoust. 2017, 123, 73-84. [CrossRef]

15. Garcia-Pozuelo, D.; Olatunbosun, O.; Strano, S.; Terzo, M. A real-time physical model for strain-based intelligent tires. Sens. Actuators A Phys. 2019, 288, 1-9. [CrossRef]

16. Khaleghian, S.; Taheri, S. Terrain classification using intelligent tire. J. Terramechanics 2017, 71, 15-24. [CrossRef]

17. Kanwar, B.S.; Saied, T. Estimation of Tire-road Friction Coefficient and its Application in Chassis Control Systems. Syst. Sci. Control. Eng. 2015, 3, 39-61.

18. Niskanen, A.; Tuononen, A.J. Three Axis IEPE Accelerometers on the Inner Liner of a Tire for Finding the Tire-Road Friction Potential Indicators. Sensors 2015, 15, 19251-19263. [CrossRef] [PubMed]

19. Hanatsuka, Y.; Higuchi, T.; Matsui, T. Development of the Road Surface Condition Classification system. In Proceedings of the IET International Conference on Information Science and Control Engineering, Shenzhen, China, 7-9 December 2012; pp. 413-416.

20. Hanatsuka, Y.; Goto, T.; Higuchi, T.; Matsui, T. Road Condition Classification using a New Global Alignment Kernel. In Proceedings of the IEEE International workshop on Machine Learning for Signal. Processing, Boston, MA, USA, 17-20 September 2015; pp. 17-20.

21. Rehman, I.U.; Nasralla, M.M.; Philip, Y. Multilayer Perceptron Neural Network-Based QoS-Aware, Content-Aware and Device-Aware QoE Prediction Model: A Proposed Prediction Model for Medical Ultrasound Streaming Over Small Cell Networks. Electronics 2019, 8, 194-221. [CrossRef]

(C) 2020 by the authors. Licensee MDPI, Basel, Switzerland. This article is an open access article distributed under the terms and conditions of the Creative Commons Attribution (CC BY) license (http://creativecommons.org/licenses/by/4.0/). 